\title{
EFECTOS DEL ESTILO DE LIDERAZGO SOBRE LA EFICACIA DE LAS ORGANIZACIONES PÚBLICAS ${ }^{1}$
}

\author{
Liliana Pedraja R. ${ }^{2} \quad$ Emilio Rodríguez P. ${ }^{3}$ \\ Recibido el 11 de marzo de 2004, aceptado el 28 de octubre de 2004
}

\begin{abstract}
RESUMEN
El presente estudio tiene como objetivo fundamental analizar la influencia del estilo o tipos de liderazgo sobre la eficacia de un conjunto de organizaciones públicas. Para este efecto, se trabaja con una muestra de 42 directivos pertenecientes a 10 instituciones públicas de la Región de Tarapacá. En este contexto, se relacionan econométricamente 3 dimensiones o estilos de liderazgo y la eficacia, construida a partir de un conjunto de factores debidamente ponderados.

Los resultados de la investigación demuestran que las organizaciones públicas presentan el estilo participativo y colaborativo en alto grado, en tanto que el estilo de liderazgo instrumental se presenta en grado medio. Por su parte, el estilo de liderazgo participativo y el estilo de liderazgo colaborativo influyen de modo positivo sobre la eficacia de las organizaciones públicas, en tanto que el estilo de liderazgo instrumental influye de manera negativa sobre la eficacia de las organizaciones públicas.
\end{abstract}

Palabras claves: Estilos de liderazgo, eficacia, organizaciones públicas.

\begin{abstract}
The main objective of the present work is to analyze the influence of leadership style on the effectiveness in a group of public organizations. This study considered a sample of 42 directors from 10 public organizations in the Tarapacá region of Chile. In this context, leadership styles, is relationship with effectiveness.

Results obtained show that these public institutions present a high degree of collaborative and participative leadership styles, whereas instrumental leadership style appears at a medium level. In addition, participative and collaborative leadership styles have a positive influence on the effectiveness of public organizations, while instrumental leadership style has a negative influence.
\end{abstract}

Keywords: Leadership style, effectiveness and public organizations.

\section{INTRODUCCIÓN}

Existen dos posiciones contrapuestas respecto de la importancia del equipo de alta dirección en la toma de decisiones estratégicas. Por un lado, la ecología de las organizaciones niega cualquier influencia del equipo de dirección sobre los resultados de la organización, pero por otro lado existen una serie de investigaciones relevantes que demuestran que ciertos aspectos relacionados al equipo de alta dirección tienen influencia sobre el proceso de toma de decisiones estratégicas y, por ende, sobre el desempeño o eficacia de la organización.
La ecología de las organizaciones propone el proceso de selección natural de las especies para las organizaciones, lo cual implica que el ambiente determinará quienes sobrevivirán y, en tal sentido, el equipo de alta dirección estará compuesto por agentes pasivos con un mínimo impacto sobre el desarrollo corporativo [24], [3], [4], [11].

Sin embargo, se plantea que los resultados de la organización, es decir, las elecciones estratégicas y el desempeño, están predeterminadas en forma parcial por las características de quienes participan de la administración [23]. Este estudio se sustenta en la premisa que en un contexto de racionalidad limitada, la base cognitiva y los valores limitan el campo de la

\footnotetext{
${ }^{1}$ Trabajo derivado de Proyecto de Investigación Mayor Universidad de Tarapacá, № 9703-01, 2001.

${ }^{2}$ Académico Departamento de Industrias. Facultad de Ingeniería. Universidad de Tarapacá, Arica-Chile, lpedraja@uta.cl

${ }^{3}$ Académico Departamento de Administración. Facultad de Ciencias, Administrativas y Económicas. Universidad de Tarapacá, Arica-Chile, erodrigu@uta.cl
} 
visión, influyendo de esta forma sobre la percepción selectiva, la interpretación y, por ende, en las percepciones de la administración y la selección de la estrategia.

En esta misma perspectiva, sin desconocer la importancia del entorno, se debe señalar que existe un conjunto de investigaciones que han demostrado que la diversidad [38], [47], [10], el estilo de liderazgo [14], [36], [50] y el tamaño del equipo de alta dirección [21], [2], constituyen variables que pueden influir sobre los procesos decisionales y por ende, sobre la eficacia de la organización.

La presente investigación se centra en descubrir si existe alguna relación estadísticamente significativa entre el estilo de liderazgo y la eficacia de la organización en las instituciones públicas de la región de Tarapacá.

\section{ASPECTOS CONCEPTUALES}

\section{El Impacto del Estilo de Liderazgo}

La teoría de los escalafones superiores se presenta como un paradigma alternativo a la ecología de las organizaciones. Su estudio comprende un conjunto de variables, pero el propósito de la presente investigación se refiere al estudio del estilo de liderazgo.

En este sentido, se plantea que es pertinente que el estilo de liderazgo sea considerado en la teoría de los escalafones superiores [13], toda vez que esta variable tiene efectos directos sobre los procesos decisionales y sobre los resultados de las organizaciones [50].

En esta misma línea de razonamiento, las investigaciones [29], [14] reafirman la idea que el estilo de liderazgo afecta los procesos de trabajo grupal, el clima social y los resultados. Se sostiene que el liderazgo participativo está más relacionado que el liderazgo directivo con el hecho de efectuar observaciones de apoyo a los miembros del grupo [29]. Por otra parte, se establece que el estilo de liderazgo influye sobre el clima y el clima influye sobre la creatividad y la productividad; además, el liderazgo influye en forma directa sobre la productividad [14].

Los estudios anteriores son consistentes [19], se plantea que los diferentes estilos de liderazgo tienen diversos efectos sobre variables tales como la flexibilidad, la responsabilidad, los estándares, las recompensas, la claridad y el compromiso y, en algunos casos, sobre el clima organizacional.
Un hecho fundamental es que el estilo de liderazgo influye sobre los subordinados [46], ya que el comportamiento del líder genera mecanismos de motivación que tienen efectos sobre la conducta de los individuos en la organización.

Por otro lado, se demuestra que el género se relaciona con el estilo de liderazgo [37] y, a su vez, éste influye sobre el estilo de decisión. Asimismo, se demuestra que el desempeño de la organización se ve influido por la cultura competitiva e innovativa [36], en tanto que el tipo de cultura se ve influenciado por el estilo de liderazgo y, por lo tanto, el estilo de liderazgo, afecta el desempeño de la organización a través de la cultura.

En forma consecuente con las investigaciones citadas [41], se realiza un estudio cuya conclusión es que el estilo de liderazgo, los procesos, los productos y los servicios, así como las personas y el enfoque a los clientes, explican los resultados de las organizaciones.

Por último, es pertinente destacar que existen diferentes estilos de liderazgo [5], [33], [28]. No obstante, es muy difícil establecer la supremacía de un determinado estilo sobre otro.

En este sentido, se postula que la definición del estilo de liderazgo frente a una determinada decisión requiere del análisis de un conjunto de factores [49], tales como: la relevancia de la decisión, la importancia del compromiso, la probabilidad de éxito, la experiencia del líder y del grupo, el apoyo del grupo al logro de los objetivos y la competencia del equipo.

La discusión anterior, apunta en la misma conclusión [50], ya que el estilo de liderazgo puede ser considerado como un determinante de la eficacia de las organizaciones.

\section{Tipología de los Estilos de Liderazgo}

Existen múltiples tipologías acerca de los estilos de liderazgo. Sin embargo, se ha determinado la importancia de distinguir 3 dimensiones del liderazgo [36]: el estilo de liderazgo participativo, el estilo de liderazgo colaborativo y el estilo de liderazgo instrumental. Obviamente, se trata de dimensiones que no son excluyentes entre sí.

El estilo de liderazgo participativo se caracteriza por cuanto el líder considera la opinión de los subordinados, y les consulta para decidir. De igual modo, cuando enfrenta un problema consulta a sus subordinados y pregunta por sugerencias. 
El estilo de liderazgo colaborativo se caracteriza porque el líder ayuda a los suyos en los trabajos encomendados, está atento al bienestar del grupo, trata a todos por igual y se afana en los detalles para que todos se sientan bien.

El estilo de liderazgo instrumental se caracteriza porque el líder explica a sus subordinados como llevar a cabo el trabajo, decide el modo en el cual las cosas se deben hacer, define los estándares de desempeño y los esquemas de trabajo, sin consulta de ningún tipo.

\section{Las Organizaciones Públicas}

Las organizaciones públicas tienen sus propias características y se diferencian de las privadas puesto que constituyen organizaciones sin fines de lucro.

En efecto, el hecho que las firmas tengan o no fines de lucro se visualiza como una característica fundamental con serias implicancias en el comportamiento estratégico de las instituciones.

Un trabajo clásico que revela un conjunto de diferencias entre las organizaciones sin fines de lucro y las organizaciones lucrativas, compara las características de las organizaciones públicas y privadas y encuentra las diferencias significativas en relación con los factores ambientales [39], la relación ambiente / organización y las estructuras internas y los procesos, todo lo cual deriva en un conjunto de implicancias estratégicas en la definición de los propósitos, los objetivos, y la planeación, en la selección de recursos humanos, administración y motivación, en el control y en la medición de los resultados.

En forma complementaria con el estudio anterior [40], se distinguen un conjunto de factores que diferencian a las organizaciones públicas, sin fines de lucro, de las organizaciones privadas, con fines de lucro. Entre tales factores destacan: la complejidad y la ambigüedad de metas, la estructura organizativa, el grado de formalización, y las actitudes y valores relativos al trabajo. Sin embargo, mediante el análisis de estudios previos, los autores descubren que los administradores de las empresas públicas consideran que tienen metas claras $\mathrm{y}$, por lo tanto no ambiguas, las cuales deben cumplir en determinados períodos de tiempo, sólo que esas metas no se refieren a maximizar el valor del patrimonio. Asimismo, los administradores de las empresas públicas no sienten que el ambiente de trabajo sea de naturaleza informal, pero sí estiman que existen procesos internos que se diferencian de las empresas privadas.
De modo similar, se realiza un meta-análisis para estudiar los resultados del cambio organizativo en el sector público, en comparación con el sector privado [42]. Los resultados indican que tanto en el sector privado como en el sector público el cambio organizativo puede resultar favorable, pero en el sector público la disposición organizativa al cambio es más lenta, producto de la estructura formal, las metas, los procedimientos administrativos y los sistemas de recompensa. Asimismo, en el sector privado la intervención que genera el cambio tiene efectos positivos sobre el comportamiento individual; en cambio, en el sector público los resultados generados por la intervención en un proceso de cambio presentan mayor variabilidad.

El punto central consiste en reconocer que las empresas sin fines de lucro presentan diferencias idiosincrásicas en relación con las firmas con fines de lucro. Sin embargo, los estudios que han dado suficiente importancia a esta situación son relativamente pocos.

Entre los estudios que han considerado, en forma debida, la diferencia entre organizaciones con y sin fines de lucro se destaca que los ejecutivos de las organizaciones lucrativas y no lucrativas ven el conflicto como indeseable [45], pero los ejecutivos de las organizaciones no lucrativas entienden que éste es necesario y que mejora la calidad de la toma de decisiones porque deja de manifiesto las necesidades de los diferentes grupos. En cambio, los ejecutivos de las organizaciones con fines de lucro subestiman la importancia del conflicto y tratan de evitarlo. Estas diferentes percepciones del conflicto se explican porque en las organizaciones no lucrativas participan múltiples intereses y los ejecutivos de estas instituciones comprenden y aceptan que dichos intereses se expresen a través del conflicto en la toma de decisiones.

De igual modo en un trabajo importante [8], en un intento por dilucidar algunas posibles condiciones para el éxito en la toma de decisiones, se encuentra que en las organizaciones lucrativas el éxito o, más bien, la eficacia de la decisión se asocia con la existencia de información y de los medios suficientes para la implantación de la decisión. En cambio, en las organizaciones no lucrativas el éxito o eficacia de la decisión se vincula con el ejercicio del derecho de las personas a participar y además, es importante la consecución de acuerdos y la ausencia de intervención por parte de la alta dirección.

Por su parte, se deja claro que existen diferencias en los procesos estratégicos entre las organizaciones no 
lucrativas y las firmas con fines de lucro [34], [9]. En efecto, se establece que la gran diferencia entre las empresas con fines de lucro y las organizaciones sin fines de lucro estriba en la forma en que ambos tipos de entidades desarrollan y evalúan su misión [34], ya que en las organizaciones no lucrativas los propósitos estratégicos escapan de la búsqueda de la rentabilidad económica. Asimismo, se sugiere que, si bien los equipos de dirección de las organizaciones no lucrativas y las firmas con fines de lucro cuentan con un razonamiento moral equivalente [9], el clima interno en unos y otros equipos difiere. De hecho en las firmas con fines de lucro, en los procesos de toma de decisiones estratégicas, los miembros del equipo de alta dirección emplean mayores niveles de racionalidad y un mayor tiempo para resolver sus decisiones, en contraste con lo que ocurre en los equipos de alta dirección en las organizaciones no lucrativas.

La comprensión de los rasgos idiosincrásicos de las organizaciones lucrativas y sin fines de lucro es una tarea esencial para medir la eficacia de este tipo de organizaciones y el impacto del estilo de liderazgo en las mismas.

En consecuencia, desde una perspectiva teórica es necesario considerar que las instituciones sin fines de lucro y, por ende, las organizaciones públicas tienen una serie de características que le hacen diferentes de las firmas privadas. Dichas características determinan el impacto de la relación entre el estilo de liderazgo y la eficacia de las organizaciones.

\section{Los Resultados de la Decisión y la Eficacia de las Organizaciones}

Los estudios que han relacionado el proceso de toma de decisiones con los resultados de las decisiones, han adoptado diferentes formas de definir y medir dichos resultados. Sin embargo, es posible clasificar la definición y medición de los resultados del proceso de toma de decisiones en tres categorías: formulación de la decisión, implantación de la decisión, y eficacia de la organización.

En relación con la formulación de la decisión, las investigaciones se centran en medir los resultados del proceso a través de la rigurosidad de la decisión, el número de alternativas generadas, la calidad de las soluciones generadas, el logro de los objetivos, el valor de la decisión, entre otros aspectos similares [26], [48], [12].
A su vez es posible encontrar investigaciones en las cuales existen tanto elementos de formulación como aspectos de implantación de las decisiones [35], [1], [8].

La implantación de la decisión es un resultado visible del proceso de toma de decisiones. Ahora bien, conjuntamente, con considerar variables tales como: el nivel de desarrollo que alcanza la decisión, el compromiso, adhesión, comprensión y aceptación afectiva que logra la decisión, y la percepción de éxito o fracaso de la decisión, resulta necesario incorporar al análisis otras variables de implantación tales como la confianza, la obediencia, la armonía social y la satisfacción por los resultados [31], [32], [44].

Por su parte, al igual que el presente trabajo, existen investigaciones que vinculan el proceso de toma de decisiones en forma directa e inmediata con la eficacia o desempeño de la organización. Entre estos estudios hay trabajos en que el desempeño o eficacia se mide a través de indicadores subjetivos, indicadores objetivos y una combinación de indicadores objetivos y subjetivos.

Como ejemplo del primero de los casos [15] se sugiere que la participación de los administradores de nivel medio en la formulación e implantación de la estrategia se relaciona en forma positiva con el desempeño de la organización. En esta investigación el desempeño de la organización se mide a través de un índice subjetivo que valora la eficacia global, la posición competitiva, la rentabilidad sobre los activos, la eficiencia de las operaciones, el desempeño financiero global, y la tasa de crecimiento.

En esta misma línea [27], se sugiere que el consenso en la toma de decisiones estratégicas tiene incidencia sobre el desempeño de la organización sólo cuando la estrategia de negocio definida es la estrategia de diferenciación. Para los investigadores el desempeño de la firma surge de la valoración subjetiva que hacen los participantes en la investigación acerca de la adaptabilidad de la firma, su eficiencia, y su eficacia.

De similar modo, se postula que un alto nivel de consenso no lleva de modo necesario a un buen desempeño de la organización [7] y en tal sentido, el consenso parece ser una condición necesaria, pero no suficiente para el éxito de la empresa. Los autores miden el desempeño de la organización de acuerdo con una valoración relativa del desempeño de la empresa en comparación con el desempeño de las firmas que operan en la misma industria respecto de la rentabilidad y las ventas. 
Por otro lado, un amplio número de investigaciones ha empleado medidas objetivas para medir el desempeño de la organización.

Por ejemplo, se concluye que el tamaño del equipo influye de modo más positivo sobre el desempeño en ambientes turbulentos que en ambientes estables [21] y que la centralización de las decisiones tiene efectos negativos sobre el desempeño de la organización. En esta investigación el desempeño de la organización se mide por la rentabilidad sobre los activos, la rentabilidad sobre las ventas, y la rentabilidad sobre el patrimonio.

De igual manera, se concluye que la heterogeneidad funcional y la heterogeneidad educacional influyen en el desempeño de la organización. Los autores establecen que el desempeño de la organización se mide por el crecimiento en la participación de mercado, y por el crecimiento en las utilidades.

Otro ejemplo en esta dirección es la investigación [20], que descubre que la racionalidad tiene efectos positivos sobre el desempeño de la organización, mientras más dinámico y menos hostil sea el ambiente. Los autores miden el desempeño de la organización por un conjunto de indicadores objetivos tales como: la rentabilidad de los activos, la rentabilidad sobre las ventas, y el volumen de ventas.

De igual modo, se encuentra que existe una relación positiva entre el grado de acuerdo en los equipos que toman decisiones y el desempeño de la organización [16]. El desempeño de la organización se mide de manera explícita por la rentabilidad sobre los activos.

Finalmente cabe mencionar que algunas investigaciones han combinado indicadores subjetivos con índices objetivos para medir el desempeño de la organización.

Por ejemplo, se construye un modelo el cual explica que las bases del comportamiento político en las organizaciones están dadas por la centralización del poder y por el conflicto [13]. Para los autores el desempeño de la organización se mide por la valoración subjetiva en escala numérica que realiza el máximo directivo acerca del desempeño de la empresa; la clasificación comparada de la valoración del desempeño de la firma en comparación con la valoración que el máximo directivo ha realizado acerca del desempeño de la competencia; y además se consideran medidas objetivas tales como el crecimiento en las ventas y la rentabilidad.
De manera similar, un modelo que determina los imperativos estratégicos para las firmas que operan en ambientes de alta velocidad [6]. La eficacia de la organización es medida a través de la valoración subjetiva que hace el principal directivo en comparación con las otras firmas del sector industrial, pero además se consideran medidas objetivas tales como las ventas y la rentabilidad.

La presente investigación construye una medición subjetiva de la eficacia, enfatizando en los rasgos idiosincrásicos de las organizaciones públicas [18], [43].

En términos específicos, se emplearon un conjunto de factores tales como: la satisfacción de los usuarios, la satisfacción de los Ministerios y Patrocinadores, la satisfacción de los funcionarios, el posicionamiento y posición relativa de la organización, y el desempeño económico, financiero y presupuestario. Respecto de cada factor se detectó su nivel de importancia y logro de tal forma de construir un índice relativo de eficacia.

\section{HIPÓTESIS DE LA INVESTIGACIÓN}

La investigación plantea las siguientes hipótesis preliminares:

H-1A: El estilo de liderazgo participativo influye de modo positivo sobre la eficacia en las organizaciones públicas.

H-1B: El estilo de liderazgo participativo influye de modo negativo sobre la eficacia en las organizaciones públicas.

H-2A: El estilo de liderazgo colaborativo influye de modo positivo sobre la eficacia en las organizaciones públicas.

H-2B: El estilo de liderazgo colaborativo influye de modo negativo sobre la eficacia en las organizaciones públicas.

H-3A: El estilo de liderazgo instrumental influye de modo positivo sobre la eficacia de las organizaciones públicas.

H-3B: El estilo de liderazgo instrumental influye de modo negativo sobre la eficacia de las organizaciones públicas. 


\section{MÉTODOS}

\section{Muestra}

Se aplica un cuestionario a 116 directivos principales de 22 organismos públicos que funcionan en la Región de Tarapacá, específicamente en la ciudad de Arica. Se intentó aplicar el cuestionario considerando una insistencia máxima de 3 veces por cada directivo. Finalmente respondieron 42 directivos de 10 organizaciones.

La tasa de respuesta asciende a un $36.2 \%$ de los directivos y a un $45.4 \%$ de las organizaciones invitadas a participar.

\section{Variables y Medidas}

Las variables y medidas empleadas para evaluar los estilos de liderazgo participativo, colaborativo e instrumental, se generaron a partir de la adaptación de ítems de las variables planteadas [36]. A su vez se empleó una escala de Likert de 1 a 7 para la medición.

A continuación se presentan los ítems referentes a cada estilo de liderazgo y a la variable de eficacia.

1. Estilo de liderazgo participativo, se preguntó si en el proceso de toma de decisiones el líder:

- Considera la opinión de los participantes.

- Considera las ideas diferencias y opiniones de los participantes.

- Pregunta su parecer a los participantes.

- Considera las sugerencias de los participantes.

- Pregunta a la totalidad de los participantes su posición.
2. Estilo de liderazgo colaborativo, se preguntó si en el proceso de toma de decisiones el líder:

- Está dispuesto a prestar colaboración a su equipo en forma permanente.

- Considera el bienestar de los participantes.

- Da un tratamiento amable e igualitario a los participantes.

- Se preocupa que el ambiente y clima de trabajo sean confortables para los miembros de su equipo.

3. Estilo de liderazgo instrumental, se consultó si en el proceso de toma de decisiones el líder:

- Decidió lo que debía hacer cada miembro del equipo.

- Decidió cómo se debían hacer las cosas en el equipo.

- Decidió los estándares esperados a lograr por el equipo.

- Definió el esquema de trabajo a realizar por los miembros del equipo.

4. Eficacia, se midió la importancia de un conjunto de factores para definir la eficacia y, posteriormente, se evaluó el nivel de logro de la organización respecto de cada factor, calculando un valor para la eficacia relativa en escala de 1 a 100 [25] y [17]. En la Tabla 1 se observan dichos factores.

\section{Validez y Fiabilidad de las Variables y Medidas}

Para medir la validez y principalmente la fiabilidad de las variables se calculó el Alpha de Cronbach, consiguiendo los resultados que se observan en la Tabla 2 .

Tabla 1. Factores de medición de eficacia.

\begin{tabular}{|c|c|c|}
\hline Factores & Grado de importancia & Nivel de logro \\
\hline Satisfacción de los usuarios. & $\begin{array}{lllll}1 & 2 & 3 & 4 & 5\end{array}$ & $\begin{array}{lllllll}1 & 2 & 3 & 4 & 5 & 6 & 7\end{array}$ \\
\hline Satisfacción de los Ministerios y patrocinadores. & $1 \quad 2 \quad 3 \quad 4 \quad 5$ & $1 \quad 2 \quad 3 \quad 4 \quad 5 \quad 6 \quad 7$ \\
\hline Satisfacción de los funcionarios. & $\begin{array}{lllll}1 & 2 & 3 & 4 & 5\end{array}$ & $\begin{array}{lllllll}1 & 2 & 3 & 4 & 5 & 6 & 7\end{array}$ \\
\hline Crecimiento, imagen y posición relativa de la organización. & $1223 \quad 4 \quad 5$ & $\begin{array}{lllllll}1 & 2 & 3 & 4 & 5 & 6 & 7\end{array}$ \\
\hline Situación económica, financiera y presupuestaria. & $\begin{array}{lllll}1 & 2 & 3 & 4 & 5\end{array}$ & $\begin{array}{lllllll}1 & 2 & 3 & 4 & 5 & 6 & 7\end{array}$ \\
\hline
\end{tabular}


Tabla 2. Validez y fiabilidad de las variables y medidas.

\begin{tabular}{|l|c|c|}
\hline \multicolumn{1}{|c|}{ Variables } & Alpha de Cronbach & Calificación de la fiabilidad \\
\hline Liderazgo participativo & 0.791 & Buena fiabilidad \\
\hline Liderazgo colaborativo & 0.665 & Dudosa fiabilidad, pero válida \\
\hline Liderazgo instrumental & 0.777 & Buena fiabilidad \\
\hline Eficacia & 0.884 & Muy buena fiabilidad \\
\hline
\end{tabular}

\section{RESULTADOS DE LA INVESTIGACIÓN}

Seguidamente se detallan los principales resultados de la investigación:

\section{Dimensiones del Estilo de Liderazgo}

Al analizar los resultados del cuestionario se obtienen los siguientes antecedentes mostrados en la Tabla 3.

Empleando la prueba estadística "t" se puede afirmar que en las organizaciones públicas objeto de estudio:

- El estilo de liderazgo participativo se presenta en un alto grado. (Ho: Media $>5.0$, test $\mathrm{t}=1.97$ ).

- El estilo de liderazgo colaborativo se presenta en un alto grado (Ho: Media $>5.0$, test $\mathrm{t}=6.48$ ).
- El estilo de liderazgo instrumental se presenta en un grado medio (Ho: Media $=3.5$, test $\mathrm{t}=1.38$ ).

\section{La Eficacia y el Estilo de Liderazgo}

Se emplea el siguiente modelo de regresión lineal múltiple:

$$
\begin{aligned}
\text { Eficacia } & =\alpha+\beta_{1} \times \text { Lid.Participativo }+ \\
& +\beta_{2} \times \text { Lid.Colaborativo }+ \\
& +\beta_{3} \times \text { Lid.Instrumental }+\mathrm{E}_{\mathrm{i}}
\end{aligned}
$$

La matriz de correlaciones de Pearson muestra los resultados descritos en la Tabla 4 .

El modelo de regresión presenta los resultados descritos en la Tabla 5 .

Tabla 3. Estilos de liderazgo.

\begin{tabular}{|l|c|c|}
\hline \multicolumn{1}{|c|}{ Variables } & Media & Desviación Estándar \\
\hline Liderazgo participativo & 5.15 & 0.494 \\
\hline Liderazgo colaborativo & 5.33 & 0.339 \\
\hline Liderazgo instrumental & 3.60 & 0.465 \\
\hline
\end{tabular}

Tabla 4. Matriz de correlaciones de Pearson.

\begin{tabular}{|l|c|c|c|c|}
\hline \multicolumn{1}{|c|}{ Variables } & Eficacia & Liderazgo Participativo & Liderazgo Colaborativo & Liderazgo Instrumental \\
\hline Eficacia & 1.000 & $0.655^{\mathrm{i}}$ & $0.437^{\mathrm{i}}$ & -0.334 ii \\
\hline Liderazgo Participativo & 0.655 & 1.000 & 0.375 & -0.217 \\
\hline Liderazgo Colaborativo & 0.437 & 0.375 & 1.000 & -0.068 \\
\hline Liderazgo Instrumental & -0.334 & -0.217 & -0.068 & 1.000 \\
\hline
\end{tabular}

i correlación significativa al $1 \%$.

ii correlación significativa al $5 \%$. 
Tabla 5. Análisis de Regresión.

\begin{tabular}{|l|c|c|c|c|}
\hline \multicolumn{1}{|c|}{ Modelo } & Beta No estandarizado & Error estándar & Test t & R cuadrado ajustado \\
\hline Constante & 16.428 & 17.973 & 0.914 & \\
\hline Lid. Participativo & 8.741 & 0.526 & $4.205^{\mathrm{i}}$ & \\
\hline Lid. Colaborativo & 5.458 & 0.226 & $1.847^{\mathrm{ii}}$ & \\
\hline Lid. Instrumental & -3.604 & -0.204 & $-1.760^{\mathrm{ii}}$ & \\
\hline R cuadrado ajustado & & & & 0.512 \\
\hline
\end{tabular}

i correlación significativa al $1 \%$.

ii correlación significativa al $10 \%$.

En consecuencia, se puede observar que:

- Existe una correlación positiva y significativa entre eficacia y liderazgo participativo y liderazgo colaborativo.

- Existe una correlación negativa y significativa entre eficacia y liderazgo instrumental.

- $\quad$ El modelo de regresión empleado explica un 51.2\% de las variaciones en la eficacia. Además no existe evidencia estadística de variables omitidas (test de la constante $=0.914$ ).

- El liderazgo participativo es una variable que influye de modo positivo en la explicación de la eficacia (test $\mathrm{t}=4.205$ ).

- El liderazgo colaborativo es una variable que influye de modo positivo en la explicación de la eficacia (test $\mathrm{t}=1.847$ ).

- El liderazgo instrumental es una variable que influye de modo negativo en la explicación de la eficacia (test $\mathrm{t}=-1.76$ ).

\section{CONCLUSIONES E IMPLICANCIAS}

La investigación permite establecer las siguientes conclusiones:

- Tanto la dimensión participativa como la colaborativa del liderazgo están presentes en alto grado en las organizaciones públicas. En tanto que el estilo de liderazgo instrumental está presente en un grado medio en las organizaciones públicas.
- El estilo de liderazgo participativo y el estilo de liderazgo colaborativo influyen de modo positivo sobre la eficacia en las organizaciones públicas.

- El estilo de liderazgo instrumental influye de modo negativo sobre la eficacia en las organizaciones públicas.

En efecto, de acuerdo a los resultados de este estudio se puede sugerir que en las organizaciones públicas,

- Se fomente la participación y se considere la opinión, sugerencias, ideas y el parecer de los participantes en el proceso decisional.

- El líder del proceso de toma de decisiones preste colaboración a su equipo, se preocupe del clima de trabajo y del bienestar del equipo y brinde a los miembros del equipo un trato amable e igualitario.

- Se evite que el líder decida siempre y por sí solo acerca de qué deben hacer los miembros de su equipo, cómo deben hacerlo, cuáles son las metas deseables y el esquema de trabajo.

\section{REFERENCIAS}

[1] A. Amason; "Distinguishing the effects of functional and dysfunctional conflict on strategic decision making: Resolving a paradox for top management teams". Academy of Management Journal, Vol. 39 N $^{\circ}$ 1, pp. 123-148, 1996.

[2] A. Amason, H. Sapienza; "The effects of top management size and interaction norms on cognitive and affective conflict". Journal of Management, Vol. 23 No 4, pp. 495-517, 1997. 
[3] T. Amburgey, H. Rao; 'Organizational ecology: Past, present and future directions". Academy of Management Journal, Vol.39, No2, pp.12651286, 1996.

[4] J. Baum, C. Oliver; "Toward an institutional ecology organizational founding". Academy of Management Journal, Vol. $39 \mathrm{~N}^{\circ} 2$, pp.1379 1427, 1996.

[5] D. Bourantas, V. Papadakis; 'Greek management". International Studies of Management \& Organization, Vol.26 No3, pp.1333, 1996.

[6] L. Bourgeois, K. Eisenhardt; 'Strategi c decision procesess in high velocity environments: Four cases in the microcomputer industry". Management Science, Vol. 34 Nº 7, pp. 816-835, 1988.

[7] C. Bowman, V. Ambrosini; 'Perceptions of strategic priorities, consensus and firm performance", Journal of Management Studies, Vol. $34 \mathrm{~N}^{\circ} 2$, pp.241-258, 1997.

[8] S. Braga, D. Hickson; 'Success in decision making: Different organizations different reasons for success". Journal of Management Studies, Vol.32 No 5, pp.655-678, 1995.

[9] H. Brower, C. Shrader; 'Moral reasoning and ethical climate: Not for profit vs for profit boards of directors". Journal of Business Ethics, Vol.26 No2, pp.147-167, 2000.

[10] M. Carpenter, J. Fredrickson; 'Top management teams, global strategy posture and moderating role of uncertainty". Academy of Management Journal, Vol.44 Nº 1, pp.533-545, 2001.

[11] E. Carroll, M. Hannan; 'The Demography of Corporations and Industries". Long Range Planning, Vol.33 № 4, pp.608, 2000.

[12] J. Dean, M. Sharfman; 'Does Decision Proce ss Matter? A Study of Strategic of Decision-making Effectiveness". Academy of Management Journal, Vol. 39 N² 2, pp. 368-396, 1996.

[13] K. Eisenhardt, L. Bourgeois; 'Politics of strategic decision making in high velocity environments: Toward a midrange theory".
Academy of Management Journal, Vol. $32 \mathrm{~N}^{\circ} 3$, pp. 543-576, 1988.

[14] G. Evkall, L. Ryhammar; 'Leadership style, social climate and organizational outcomes: A study of a Swedish University College". Creativity and Innovation Management, Vol. 7 No3, pp.126-130, 1997.

[15] S. Floyd, B. Wooldridge; 'Middle management strategic influence and organizational performance". Journal of Management Studies, Vol. 34 No3, pp.465-485, 1997.

[16] J. Fredrickson, A. Iaquinto; 'Inertia and creeping rationality in strategic decision processes". Academy of Management Journal, Vol. $32 \mathrm{~N}^{\circ} 4$, pp. 516-542, 1989.

[17] L. Guerras, J. García-Tenorio y M. Pérez; 'El Papel de las Unidades Estratégicas de Negocio en el Proceso de Dirección Estratégica de la Empresa". Boletín de Estudios Económicos, Vol. $49 \mathrm{~N}^{\mathrm{o}} 152$, pp.239-256, 1994.

[18] L. Guerras; 'La dirección estratégica en las organizaciones no lucrativas", Civitas Madrid, 1995, pp. 457-472.

[19] D. Goleman; 'Leadership that gets results". Harvard Business Review, Vol.78 N², pp.78-90, 2000.

[20] I. Goll, A. Rashed; 'Rational decision-making and firm performance: The moderating role of environment". Strategic Management Journal, Vol. 18, pp. 583-591, 1997.

[21] J. Haleblian, S. Finkelstein; 'Top management team size, CEO dominance and firm performance". Academy of Management Journal, Vol.36 No 4, pp.844863, 1993.

[22] D. Hambrick, T. Cho y M. Chen; "The influence of top management team heterogeneity on firm's competitive moves", Administrative Science Quarterly, Vol. 41, 1996, pp.659-684.

[23] D. Hambrick, P. Mason; 'Upper echelons: The organization as a reflection of its top managers". Academy of Management Review, Vol.9 № 2, pp.193-206, 1984. 
[24] M. Hannan, J. Freeman; 'The population ecology of organizations". Americ an Journal of Sociology, Vol.82 № 5, pp.929-963, 1977.

[25] K. Hatten, M. Hatten; 'Strategic groups, asymmetrical mobility barriers and contestability". Strategic Management Journal, Vol. 8, pp.329-342, 1987.

[26] J. Hollenbeck, D. Ilgen, J. LePine, J. Colquitt y J. Hedlund; 'Extending the multilevel theory of team decision making: Effects of feedback and experience in hierarchical teams". Academy of Management Journal, Vol.41, pp.269-282, 1998.

[27] C. Homburg, H. Krohmer, J. Workman; 'Strategic consen sus and performance: The role of strategy type and market related dynamism". Strategic Management Journal, Vol. 20, pp. 339357, 1999.

[28] O. Ingers; 'Viewpoint: A leader's inner security might be a crucial factor for choice of leadership style". Journal of European Industrial Training, Vol.19 No 4, pp.19-20, 1995.

[29] S. Kahai, J. Sosik; 'Effects of leadership style and follwers' cultural orientation on performance in group and individual task conditions". Personnel Psychology, Vol.50 No 1, pp.121-147, 1997.

[30] W. Kim, R. Maugborne; 'Implementing global strategies: The role of procedural justice". Strategic Management Journal, Vol. 12, pp. 125143, 1991.

[31] W. Kim, R. Maugborne; 'Procedural justice attitudes, and subsidiary top management compliance with multinational' $\mathrm{s}$ corporate strategic decisions". Academy of Management Journal, Vol. $36 \mathrm{~N}^{\circ} 3$, pp. 502-526, 1993.

[32] W. Kim, R. Maugborne; 'Making global strategies work". Sloan Management Review, Spring, pp. 11-27, 1993.

[33] K. Lowe, G. Galen; "Effectiveness correlates of transformational and transactional leadership: A meta-analytic review of MLQ literature", Leadership Quarterly, Vol.7 №3, pp.385-426, 1996.
[34] Mc Farlan; 'Working in nonprofit boards: Don't assume the shoe fits". Harvard B usiness Review, Vol.77 Nº, pp. 6480, 1999.

[35] P. Nutt; 'Framing strategic decisions". Organization Science, Vol. $9 \mathrm{~N}^{\mathrm{o}} 2$, pp. 195-216, 1998.

[36] E. Ogbonna, L. Harris; 'Leadership style, organizational culture and performance: Empirical evidence from U.K. companies". International Journal of Human Resource Management, Vol.11 No 4, pp.766-788, 2000.

[37] D. Park; 'Gender role, decision style and leadership style". Women in Management Review, Vol.11 № 8, pp.13-17, 1996.

[38] L. Pelled, K. Eisenhardt y K. Xin; 'Exploring the black box: An analysis of work group diversity, conflict, and performance". Administrative Science Quarterly, Vol. 44 Nº 1, pp. 1-28, 1999.

[39] H. Rainey, R. Backoff y C. Levine; 'Comparing public and private organizations". Public Administration Review, Vol.32 N², pp.233-244, 1976.

[40] H. Rainey, B. Bozeman; 'Comparing public and private organizations: Empirical research and power of the a priori". Journal of Public Research and Theory, Vol.10 N², pp.447-469, 2000 .

[41] S. Rahman; "Total quality management practices and business outcome: Evidence from small and medium enterprises in Western Australia". Total Quality Management, Vol.12 No2, pp.201-210, 2001.

[42] P. Robertson, S. Seneviratne; 'Outcomes of planned organizational change in the public sector: A meta-analytic comparison to the private sector". Public Administration Review, Vol.55 $\mathrm{N}^{\mathrm{o}} 6$, pp.547-558, 1995.

[43] E. Rodríguez; 'Determinantes y efectos de la toma de decisiones estratégicas: Evidencia empírica en las instituciones universitarias de España y Chile". Tesis Doctoral, Universidad Complutense de Madrid, 2002. 
[44] H. Sapienza, M. Korsgaard; "Procedural justice in entrepreneur-investor relations". Academy of Management Journal, Vol. $39 \mathrm{~N}^{\circ} 3$, pp. 544574, 1996.

[45] C. Schwenk; "Conflict in organizational decision making: An exploratory study of its effects in for profit and not for profit organizations". Management Science, Vol.36 No 4, pp.436-448, 1990.

[46] B. Shamir, R. House y M. Arthur; 'The motivational effects of charismatic leadership: A self-concept based theory". Organization Science, Vol.4 No 4, pp.577-594, 1993.

[47] T. Simonds, L. Pelled y K. Smith; 'Making use of difference: Diversity, debate and decision comprehensiveness in top management teams". Academy of Management Journal, Vol.42 $\mathrm{N}^{\circ} 6$, pp. 662-673, 1999.
[48] J. Valacich, C. Schwenk; 'Structuring conflict in individual, face-to-face, and computer-mediated group decision making: Carping versus objective devil' s advocacy”. Decision Science, Vol. 26 No 3, pp. 369-393, 1995.

[49] V. Vroom; 'Leadership and decision making process", Organizational Dynamics, Vol.28 No 4 , pp.82-94, 2000.

[50] D. Waldman, G. Ramírez, R. House y P. Puranam; 'Does leadership matter, CEO leadership attributes and profitability under conditions of perceived environmental uncertainty". Academy of Management Journal, Vol.38 No 4, pp.943-974, 2001. 